\title{
Front Matter: Volume 6808
}

, "Front Matter: Volume 6808," Proc. SPIE 6808, Image Quality and System Performance V, 680801 (28 January 2008); doi: 10.1117/12.790443

SPIE. Event: Electronic Imaging, 2008, San Jose, California, United States 


\title{
PROCEEDINGS Electronic Imaging \\ Sclence and Tochnology
}

\section{Image Quality and System Performance V}

\author{
Susan P. Farnand \\ Frans Gaykema \\ Editors
}

28-30 January 2008

San Jose, California, USA

Sponsored and Published by

IS\&T-The Society for Imaging Science and Technology

SPIE 
The papers included in this volume were part of the technical conference cited on the cover and title page. Papers were selected and subject to review by the editors and conference program committee. Some conference presentations may not be available for publication. The papers published in these proceedings reflect the work and thoughts of the authors and are published herein as submitted. The publishers are not responsible for the validity of the information or for any outcomes resulting from reliance thereon.

Please use the following format to cite material from this book:

Author(s), "Title of Paper," in Image Quality and System Performance V, edited by Susan P. Farnand, Frans Gaykema, Proceedings of SPIE-IS\&T Electronic Imaging, SPIE Vol. 6808, Article CID Number (2008).

\author{
ISSN 0277-786X \\ ISBN 9780819469809
}

\author{
Copublished by \\ SPIE \\ P.O. Box 10, Bellingham, Washington $98227-0010$ USA \\ Telephone +1 3606763290 (Pacific Time) · Fax +1 3606471445 \\ SPIE.org \\ and \\ IS\&T-The Society for Imaging Science and Technology \\ 7003 Kilworth Lane, Springfield, Virginia, 22151 USA \\ Telephone +1 7036429090 (Eastern Time) · Fax +1 7036429094 \\ imaging.org
}

Copyright (c) 2008, Society of Photo-Optical Instrumentation Engineers and The Society for Imaging Science and Technology.

Copying of material in this book for internal or personal use, or for the internal or personal use of specific clients, beyond the fair use provisions granted by the U.S. Copyright Law is authorized by the publishers subject to payment of copying fees. The Transactional Reporting Service base fee for this volume is $\$ 18.00$ per article (or portion thereof), which should be paid directly to the Copyright Clearance Center (CCC), 222 Rosewood Drive, Danvers, MA 01923. Payment may also be made electronically through CCC Online at copyright.com. Other copying for republication, resale, advertising or promotion, or any form of systematic or multiple reproduction of any material in this book is prohibited except with permission in writing from the publisher. The CCC fee code is 0277-786X/08/ $\$ 18.00$.

Printed in the United States of America.

Paper Numbering: Proceedings of SPIE follow an e-First publication model, with papers published first online and then in print and on CD-ROM. Papers are published as they are submitted and meet publication criteria. A unique, consistent, permanent citation identifier (CID) number is assigned to each article at the time of the first publication. Utilization of CIDs allows articles to be fully citable as soon they are published online, and connects the same identifier to all online, print, and electronic versions of the publication. SPIE uses a six-digit CID article numbering system in which:

- The first four digits correspond to the SPIE volume number.

- The last two digits indicate publication order within the volume using a Base 36 numbering system employing both numerals and letters. These two-number sets start with 00, 01, 02, 03, 04, 05, 06, 07, 08 , $09,0 A, O B \ldots O Z$, followed by 10-1Z, 20-2Z, etc.

The CID number appears on each page of the manuscript. The complete citation is used on the first page, and an abbreviated version on subsequent pages. Numbers in the index correspond to the last two digits of the six-digit CID number. 


\section{Contents}

ix Conference Committee

\section{SESSION 1 IMAGE QUALITY STANDARDS FOR CAPTURE, PRINT, AND DISPLAY}

680802 INCITS W1.1 development update: appearance-based image quality standards for printers (Invited Paper) [6808-01]

E. K. Zeise, Eastman Kodak Co. (USA); D. R. Rasmussen, Xerox Corp. (USA); Y. S. Ng, Eastman Kodak Co. (USA); E. Dalal, Xerox Corp. (USA); A. McCarthy, Lexmark Corp. (USA);

D. Williams, Image Science Associates (USA)

680803 Characterization of reflection scanner uniformity [6808-02]

E. K. Zeise, Eastman Kodak Co. (USA); W. C. Kress, Toshiba America Information Systems, Inc. (USA); D. R. Williams, Image Science Associates (USA)

680804 A pilot study of digital camera resolution metrology protocols proposed under ISO 12233 , edition 2 [6808-03]

D. Williams, Image Science Associates (USA); D. Wueller, Image Engineering (USA);

K. Matherson, Hewlett Packard (USA); H. Yoshida, Olympus Imaging Corp. (USA); P. Hubel, Foveon, Inc. (USA)

680805 Sampling efficiency in digital camera performance standards [6808-04]

P. D. Burns, Carestream Health, Inc. (USA); D. Williams, Image Science Associates (USA)

\section{SESSION 2 IMAGE QUALITY ATTRIBUTES CHARACTERIZATION AND MEASUREMENT: PRINTER}

680806 Evaluation of characterization methods of printer MTF [6808-05]

A. J. Lindner, N. Bonnier, Télécom Paris (France) and OCE-PLT (France); C. Leynadier, OCE-PLT (France); F. Schmitt, Télécom Paris (France)

680807 Printer resolution measurement based on slanted edge method [6808-06]

Y. Bang, S. H. Kim, D. C. Choi, Samsung Electronics Co. Ltd. (South Korea)

680808 Robust estimation of print mottle [6808-07]

Z. Fan, W. Wu, E. N. Dalal, R. Rasmussen, Xerox Corp. (USA)

680809 Characterization of mottle and low-frequency print defects [6808-09]

A. H. Eid, B. E. Cooper, E. E. Rippetoe, Lexmark International Inc. (USA)

6808 OA Development of softcopy environment for primary color banding visibility assessment [6808-10]

B. Min, Z. Pizlo, J. P. Allebach, Purdue Univ. (USA)

$6808 \mathrm{OB}$ On estimation of perceived mottling prior to printing [6808-50]

A. Sadovnikov, L. Lensu, H. Kälviäinen, Lappeenranta Univ. of Technology (Finland) 
6808 0C Motion blur perception in various conditions of presented edge [6808-11]

S. Nakagawa, T. Nakaguchi, N. Tsumura, Y. Miyake, Chiba Univ. (Japan)

6808 OD Noise estimation from a single image taken by specific digital camera using a priori information [6808-12]

H. Ito, K. Kamimura, N. Tsumura, T. Nakaguchi, Chiba Univ. (Japan); H. Motomura, Matsushita Electric Industrial Co., Ltd. (Japan); Y. Miyake, Chiba Univ. (Japan)

$6808 \mathrm{OE} \quad$ Matching image color from different cameras [6808-13]

M. D. Fairchild, D. R. Wyble, RIT (USA); G. M. Johnson, Apple, Inc. (USA)

6808 OF Predicting image quality using a modular image difference model [6808-14]

M. Orfanidou, S. Triantaphillidou, E. Allen, Univ. of Westminster (United Kingdom)

$68080 \mathrm{G}$ Hand motion and image stabilization in hand-held devices [6808-44]

E. Mar-Or, P. Dmitry, Advasense Technologies Ltd. (Israel)

$6808 \mathrm{OH} \quad$ Visual quality metric using one-dimensional histograms of motion vectors [6808-52]

H.-S. Han, D.-O Kim, R.-H. Park, Sogang Univ. (South Korea); D.-G. Sim, Kwangwoon Univ. (South Korea)

\section{SESSION 4 SUBJECTIVE IMAGE QUALITY EVALUATION METHODOLOGY}

6808 Ol Perceptual color difference metric including a CSF based on the perception threshold [6808-15]

V. Rosselli, M.-C. Larabi, C. Fernandez-Maloigne, Univ. of Poitiers (France)

6808 0J Anchored paired comparisons [6808-16]

E. N. Dalal, J. C. Handley, W. Wu, J. Wang, Xerox Corp. (USA)

6808 OK Online image quality surveys based on response time [6808-18]

D. R. Rasmussen, Xerox Corp. (USA)

$6808 \mathrm{OL} \quad$ Framework for modeling visual printed image quality from the paper perspective [6808-17] P. Oittinen, R. Halonen, A. Kokkonen, Helsinki Univ. of Technology (Finland); T. Leisti, G. Nyman, Univ. of Helsinki (Finland); T. Eerola, L. Lensu, H. Kälviäinen, Lappeenranta Univ. of Technology (Finland); R. Ritala, J. Pulla, M. Mettänen, Tampere Univ. of Technology (Finland)

$68080 \mathrm{M} \quad$ Forming valid scales for subjective video quality measurement based on a hybrid qualitative/quantitative methodology [6808-19]

T. Virtanen, J. Radun, P. Lindroos, S. Suomi, T. Säämänen, Univ. of Helsinki (Finland); T. Vuori, M. Vaahteranoksa, Nokia Technology Platforms (Finland); G. Nyman, Univ. of Helsinki (Finland)

$68080 \mathrm{~N}$ Measuring multivariate subjective image quality for still and video cameras and image processing system components [6808-20]

G. Nyman, T. Leisti, P. Lindroos, J. Radun, S. Suomi, T. Virtanen, Univ. of Helsinki (Finland); J.-L. Olives, J. Oja, T. Vuori, Nokia Research Ctr. (Finland) 
680800 Visual experiments on the web: design of a web-based visual experiment management system [6808-48]

S. Zuffi, E. Beltrame, P. Scala, ITC, CNR (Italy)

\section{SESSION 5 IMAGE QUALITY EVALUATION CONCEPTS}

6808 OP Process perspective on image quality evaluation [6808-22]

T. Leisti, Univ. of Helsinki (Finland); R. Halonen, A. Kokkonen, Helsinki Univ. of Technology (Finland); H. Weckman, Univ. of Helsinki (Finland); M. Mettänen, Tampere Univ. of Technology (Finland); L. Lensu, Lappeenranta Univ. of Technology (Finland); R. Ritala, Tampere Univ. of Technology (Finland); P. Oittinen, Helsinki Univ. of Technology (Finland); G. Nyman, Univ. of Helsinki (Finland)

$6808 \mathrm{OQ}$ Are existing procedures enough? Image and video quality assessment: review of subjective and objective metrics [6808-53]

S. Ouni, Univ. de Reims Champagne-Ardenne (France) and Institut Supérieur d'Informatique (Tunisia); M. Chambah, M. Herbin, Univ. de Reims Champagne-Ardenne (France); E. Zagrouba, Institut Supérieur d'Informatique (Tunisia)

6808 OR Digital image improvement by adding noise: an example by a professional photographer [6808-49]

T. Kurihara, Y. Manabe, N. Aoki, H. Kobayashi, Chiba Univ. (Japan)

\section{SESSION 6 SYSTEMS PERFORMANCE: MODELING}

6808 OS Image quality evaluation using generalized natural image [6808-23]

K. Kagitani, Ricoh Co., Ltd. (Japan)

6808 OT Toward an efficient objective metric based on perceptual criteria [6808-24]

L. Quintard, Univ. of Poitiers (France) and Lab. national de métrologie et d'essais (France); M.-C. Larabi, C. Fernandez-Maloigne, Univ. of Poitiers (France)

6808 OU A color image quality assessment using a reduced-reference image machine learning expert [6808-25]

C. Charrier, G. Lebrun, O. Lezoray, Univ. de Caen Basse-Normandie, CNRS (France)

6808 0V Data path design and image quality aspects of the next generation multifunctional printer [6808-26]

M. H. H. Brassé, S. P. R. C. de Smet, Océ Technologies B.V. (Netherlands)

\section{SESSION 7 SYSTEMS PERFORMANCE: VIDEO AND DISPLAY I}

6808 OW DAF: differential ACE filtering image quality assessment by automatic color equalization [6808-27]

S. Ouni, M. Chambah, Univ. de Reims Champagne Ardenne (France); C. Saint-Jean, Univ. of La Rochelle (France); A. Rizzi, Univ. of Milano (Italy) 
6808 0X No-reference method for image effective bandwidth estimation [6808-28]

B. Fishbain, L. Yaroslavsky, I. Ideses, Tel Aviv Univ. (Israel); F. Roffet-Crété, STMicroelectronics (France)

6808 OY Colour analysis and verification of CCTV images under different lighting conditions [6808-29]

R. A. Smith, K. MacLennan-Brown, Home Office Scientific Development Branch (United Kingdom) and Univ. of Westminster (United Kingdom); J. F. Tighe, N. Cohen, Home Office Scientific Development Branch (United Kingdom); S. Triantaphillidou, Univ. of Westminster (United Kingdom); L. W. MacDonald, Univ. of the Arts London (United Kingdom)

$68080 Z$ The relationship between preferred luminance and TV screen size [6808-31]

T. Fujine, Y. Yoshida, M. Sugino, Sharp Corp. (Japan)

\section{SESSION 8 SYSTEMS PERFORMANCE: VIDEO AND DISPLAY II}

680810 Increasing display performance over a wide range of viewing angles by means of improved calibration algorithms [6808-34]

T. Kimpe, G. Van Hoey, Barco (Belgium)

680811 An image similarity measure using homogeneity regions and structure [6808-35] E. P. Lam, K. C. Loo, Thales Raytheon Systems (USA)

680812 Internet-based assessment of image sharpness enhancement [6808-36] L. MacDonald, London College of Communication (United Kingdom); S. Bouzit, St. Andrew's Univ. (United Kingdom)

680813 Autonomously detecting the defective pixels in an imaging sensor array using a robust statistical technique [6808-37]

S. Ghosh, Univ. of Kent (United Kingdom); I. Marshall, Lancaster Univ. (United Kingdom);

A. Freitas, Univ. of Kent (United Kingdom)

680814 Super-resolution image reconstruction from UAS surveillance video through affine invariant interest point-based motion estimation [6808-45]

Q. He, Mississippi Valley State Univ. (USA); R. R. Schultz, Y. Wang, A. Camargo, F. Martel, Univ. of North Dakota (USA)

680815 Relation between bitrate, motion, and framerate for scoring of image sequences [6808-51] M.-C. Larabi, L. Quoirin, Univ. de Poitiers (France)

\section{SESSION 9 CONTEXT-DEPENDENT IMAGE EVALUATION}

680816 The flux: creating a large annotated image database [6808-38]

D. Tamburrino, P. Schönmann, P. Vandewalle, S. Süsstrunk, Ecole Polytechnique Fédérale de Lausanne (Switzerland)

680817 Improving holiday pictures: winter and beach image enhancement [6808-39]

L. Marchesotti, M. Bressan, Xerox Research Ctr. Europe (France) 
680818 Megapixel mythology and photospace: estimating photospace for camera phones from large image sets [6808-40]

B. O. Hultgren, Image Integration, Inc. (USA); D. W. Hertel, Sensata Technologies, Inc. (USA)

\section{SESSION 10 EMERGING TECHNOLOGIES: 3D VIDEO AND PRINT}

680819 Performance evaluation of 3D-TV systems (Invited Paper) [6808-41]

R. G. Kaptein, Philips Research Labs. (Netherlands); A. Kuijsters, M. T. M. Lambooij, Philips Research Labs. (Netherlands) and Eindhoven Univ. of Technology (Netherlands);

W. A. IJsselsteijn, Eindhoven Univ. of Technology (Netherlands); I. Heynderickx, Philips

Research Labs. (Netherlands) and Delft Univ. of Technology (Netherlands)

6808 1A The effect of added dimensionality on perceived image value [6808-42]

S. Farnand, Rochester Institute of Technology (USA)

6808 1B Measuring stereoscopic image quality experience with interpretation-based quality methodology [6808-43]

J. Häkkinen, Univ. of Helsinki (Finland) and Nokia Research Ctr. (Finland); T. Kawai, Waseda Univ. (Japan); J. Takatalo, T. Leisti, J. Radun, A. Hirsaho, G. Nyman, Univ. of Helsinki (Finland)

Author Index 
Downloaded From: https://www.spiedigitallibrary.org/conference-proceedings-of-spie on 26 Apr 2023

Terms of Use: https://www.spiedigitallibrary.org/terms-of-use 


\title{
Conference Committee
}

\author{
Symposium Chair
}

Nitin Sampat, Rochester Institute of Technology (USA)

Conference Chairs

Susan P. Farnand, Rochester Institute of Technology (USA)

Frans Gaykema, Océ Technologies BV (Netherlands)

Program Committee

Peter D. Burns, Carestream Health, Inc. (USA)

Majed Chambah, Université de Reims Champagne-Ardenne (France)

Luke C. Cui, Lexmark International, Inc. (USA)

Mark D. Fairchild, Rochester Institute of Technology (USA)

Dirk W. Hertel, Sensata (USA)

Robin Jenkin, Rhevision Technology, Inc. (USA)

Sang Ho Kim, Samsung Electronics Company, Ltd. (South Korea)

Lindsay W. MacDonald, University of the Arts London (United Kingdom)

Yoichi Miyake, Chiba University (Japan)

Nathan Moroney, Hewlett-Packard Company (USA)

Göte S. Nyman, University of Helsinki (Finland)

D. René Rasmussen, Xerox Corporation (USA)

Sophie Triantaphillidou, University of Westminster (United Kingdom)

Eric K. Zeise, Eastman Kodak Company (USA)

Session Chairs

$1 \quad$ Image Quality Standards for Capture, Print, and Display

Susan P. Farnand, Rochester Institute of Technology (USA)

2 Image Quality Attributes Characterization and Measurement: Printer Eric K. Zeise, Eastman Kodak Company (USA)

3 Image Quality Attributes Characterization and Measurement:

Capture and Display

Peter D. Burns, Carestream Health, Inc. (USA)

$4 \quad$ Subjective Image Quality Evaluation Methodology

Mark D. Fairchild, Rochester Institute of Technology (USA) 
$5 \quad$ Image Quality Evaluation Concepts

Sophie Triantaphillidou, University of Westminster (United Kingdom)

$6 \quad$ Systems Performance: Modeling

D. René Rasmussen, Xerox Corporation (USA)

$7 \quad$ Systems Performance: Video and Display I

Robin B. Jenkin, Rhevision Technology, Inc. (USA)

8 Systems Performance: Video and Display II

Göte S. Nyman, University of Helsinki (Finland)

9 Context-dependent Image Evaluation

Luke C. Cui, Lexmark International, Inc. (USA)

10 Emerging Technologies: 3D Video and Print

Frans Gaykema, Océ Technologies B.V. (Netherlands) 